\title{
WDM packet switch architectures and analysis of the influence of tunable wavelength converters on the performance
}

\author{
Danielsen, Søren Lykke; Mikkelsen, Benny; Jørgensen, Carsten; Durhuus, Terji; Stubkjær, Kristian
}

Published in:

Journal of Lightwave Technology

Link to article, DOI:

$10.1109 / 50.554327$

Publication date:

1997

Document Version

Publisher's PDF, also known as Version of record

Link back to DTU Orbit

Citation (APA):

Danielsen, S. L., Mikkelsen, B., Jørgensen, C., Durhuus, T., \& Stubkjær, K. (1997). WDM packet switch architectures and analysis of the influence of tunable wavelength converters on the performance. Journal of Lightwave Technology, 15(2), 219 - 227. https://doi.org/10.1109/50.554327

\section{General rights}

Copyright and moral rights for the publications made accessible in the public portal are retained by the authors and/or other copyright owners and it is a condition of accessing publications that users recognise and abide by the legal requirements associated with these rights.

- Users may download and print one copy of any publication from the public portal for the purpose of private study or research.

- You may not further distribute the material or use it for any profit-making activity or commercial gain

- You may freely distribute the URL identifying the publication in the public portal 


\title{
WDM Packet Switch Architectures and Analysis of the Influence of Tuneable Wavelength Converters on the Performance
}

\author{
Soeren Lykke Danielsen, Benny Mikkelsen, Carsten Joergensen, Terji Durhuus, \\ and Kristian E. Stubkjaer, Member, IEEE
}

\begin{abstract}
A detailed analytical traffic model for a photonic wavelength division multiplexing (WDM) packet switch block is presented and the requirements to the buffer size is analyzed. Three different switch architectures are considered, each of them representing different complexities in terms of component count and requirements to the components. It is shown that the number of fiber delay-lines, that form the optical buffer, can be substantially reduced by the use of tuneable optical wavelength converters, thereby exploiting the wavelength domain to solve contention of optical packets. For a $16 \times 16$ switch with four wavelength channels per inlet, all at a load of 0.8 , the number of delay-lines is reduced from 47 to 12 by use of tuneable optical wavelength converters. Apart from the number of delay-lines the physical buffer structure is analyzed with special attention to the possibilities offered by optics, i.e., the possibility of several outlets sharing the same physical buffer. For the three architectures presented here, a tradeoff in the buffer architectures is addressed: a buffer physically shared among all outlets requires many wavelengths internally in the switch block, whereas, architectures with buffers dedicated to each outlet require a smaller number of wavelengths.
\end{abstract}

Index Terms-Optical buffering, packet switching, wavelength conversion, WDM networks.

\section{INTRODUCTION}

W AVELENGTH division multiplexing (WDM) has been proposed as a way of increasing the capacity in future optical networks [1], [2]. Furthermore, WDM networks are likely to use optical switching to avoid the bottlenecks of electronics [3] as well as to increase the transparency of optical networks [4]. Additionally, as the asynchronous transfer mode (ATM) has been promoted as the standard in B-ISDN, the study of optical packet switching is of great importance [5]-[7]. However, the absence of an effective way to store cells in the optical domain, reduces the realistic buffer capacity of optical packet switches. As an example, a cell loss probability (CLR) of $10^{-10}$ at a load of 0.8 of random traffic requires a buffer capacity of 47 cells per switch outlet for a $16 \times 16$ switch [8]. Realising the optical buffer by fiber delay-lines, as proposed in [5], requires lengths up to $1.6 \mathrm{~km}$ at $2.5 \mathrm{~Gb} / \mathrm{s}$. This is of concern for reasons of complexity as well as compactness.

In this paper we show, that by exploiting the wavelength dimension by using WDM and employing tuneable optical

Manuscript received December 11, 1995; revised October 7, 1996.

The authors are with the Electromagnetics Institute, Technical University of Denmark, DK-2800 Lyngby, Denmark.

Publisher Item Identifier S 0733-8724(97)01379-0. wavelength converters (TOWC's) the required number of fiber delay-lines in optical packet switches can be reduced. To analyze the influence of using the wavelength domain we derive a traffic model based on the Markov chain analysis described in [8] and which is a detailed description of the model used in [9]. The use of WDM in optical packet switching can to some extent be compared to using multichannel transmission groups in electronic ATM switching as described in [10]. However, when using the wavelength dimension in the optical domain the sequence of cells belonging to the same connection may be disturbed. This will cause the cells to arrive in a different order than they were transmitted thereby breaking the cell integrity rule. Therefore, to fully describe the packet switching performance in terms of cell loss we take this phenomenon into account.

It is emphasised, that the objective of this paper is not to perform a detailed traffic analysis but to illustrate how wavelength converters can relax the requirements to the buffer dimension and thereby reduce the switch complexity of optical packet switches. Furthermore, we show that the wavelength dimension allows a high degree of freedom in terms of constructing the switch blocks needed for optical packet switching.

The paper is organized as follows. We begin by describing the network to be considered. Next, three different architectures for the optical packet switch are proposed and discussed with special attention to the buffer size, switch complexity and the required number of wavelengths. Next, the traffic model is derived and verified by simulations. The model is used to compare the switch block performances for the switch blocks with and without tuneable wavelength converters. Moreover, the complexity of the three proposed architectures is compared with respect to the component count and the requirements to the optical devices.

\section{NETWork AND SWITCH Description}

The general optical WDM network which we consider is shown in Fig. 1. It is a mesh network in which signals on $n$ different wavelengths, $\lambda_{1} \cdots \lambda_{n}$, are used to carry cell traffic between the optical network nodes. A possible realization of one such node architecture is given in Fig. 2(a) (configuration a). The switch consists of three main blocks: 1) the cell encoder where a demultiplexer (DMUX) selects the cells arriving at the $n$ fixed wavelengths, $\lambda_{1} \cdots \lambda_{n}$, and TOWC's address free space in the fiber delay-line output buffers; 2 ) 




Fig. 1. General WDM network where $n$ wavelength channels are transmitted between each switch block.

a nonblocking space-switch to access the desired outlet as well as appropriate delay-line in the output buffer; 3) cell buffers, that are realized by fiber delay-lines. Furthermore, at the output of the switch, multipassband filters are inserted to reduce the possible noise generated by the devices within the switch block. As seen from the figure, the size of the spaceswitch is $N x n \cdot N(B / n+1)$ where $B$ is the number of cell positions in the buffer, $n$ the number of wavelengths, $N$ the number of in- and out-lets and $B / n$ the number of delaylines. Note that the last of the $B / n+1$ connections from the space switch through the buffer to each outlet is a fiber with an infinitesimal length, i.e., this connection is not a delayline. In the following we denote the term $B / n+1$ as, $\alpha$, for simplicity and stress that for a given number of fiber delaylines the number of cell positions, $B$, is a multiplum of $n$ so that $\alpha$ is an integer.

As shown in Fig. 2, there are other possible architectures for realizing the optical packet switch. The architecture in Fig. 2(b) (configuration $b$ ) reduces the total space-switch size to $N x n \cdot \alpha$ by physically sharing one buffer, but the number of wavelengths required internally in the switch is increased from $n$ to $n \cdot N$. In addition to this, converters that convert to fixed wavelengths $\left(\lambda_{1} \cdots \lambda_{n}\right)$ are needed at the output of the switch block for the cells to be transmitted on the same $n\left(\lambda_{1} \cdots \lambda_{n}\right)$ wavelength channels that are used at the input. To reduce the number of wavelengths internally in the switch, an architecture as shown in Fig. 2(c) (configuration $c$ ) could be implemented. Here, $n$ physical buffers are shared among all of the outlets, but the cells from each buffer is restricted to go to $N$ of the $n N$ internal switch outlets, so that no wavelength blocking occurs. With configuration $c$, the number of wavelengths is reduced from $n \cdot N$ to $N$ while the total size of the spaceswitch is increased from $N x n \cdot \alpha$ to $N x n \cdot n \cdot \alpha$ compared to configuration $b$.

Not shown in the three architectures are optical to electrical interfaces situated just after the demultiplexers at the switch inlets. These interfaces are used to extract the header of each packet by which the destination and thereby switch outlet can be found. With this information as well as knowledge of the queueing situation, the output wavelength of the converters

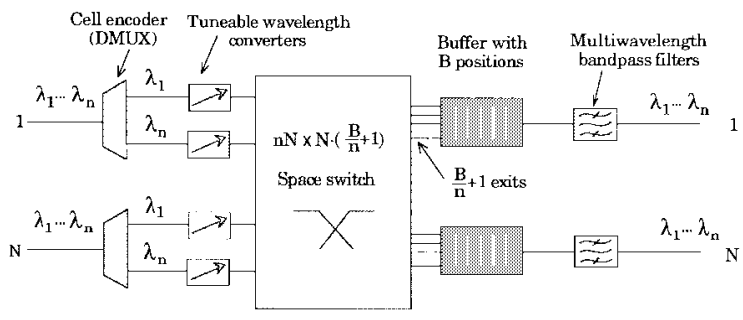

(a)



(b)



(c)

Fig. 2. (a) WDM ATM switch block with buffers realized by fiber delay-lines and with tuneable wavelength converters to address free space in the buffers. (b) WDM ATM switch configuration where $n \cdot N$ internal wavelengths are used to perform the queuing operation in the physically shared buffer. (c) Switch configuration where $N$ internal wavelengths are needed to perform the queuing operation in the $n$ physically shared buffers.

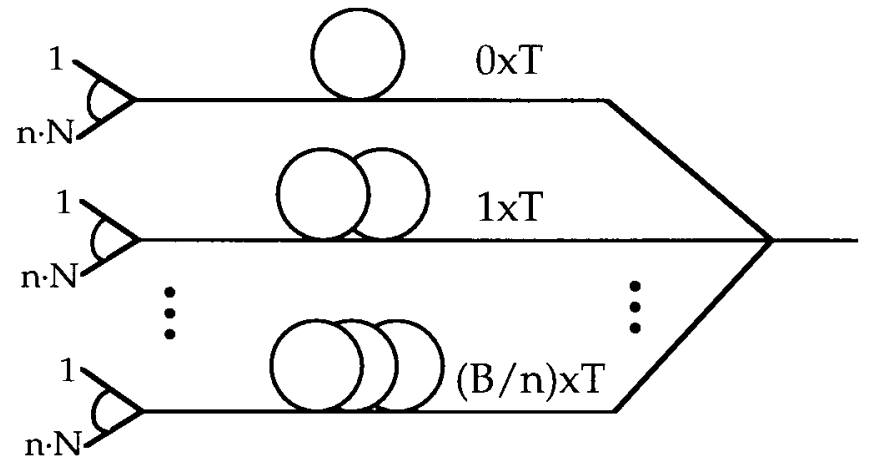

Fig. 3. Physical layout of fiber delay-line buffer architecture. $B$ is the number of cell positions in the buffer, $n$ the number of wavelength channels per in- and out-let, $N$ is the number of physical in- and out-lets while $T$ is the duration of a cell

as well as state of the gates within the space switch can be electrically controlled. This operation is the same for all the presented architectures and consequently the size of the buffers, $\alpha$, is the same for the three configurations. The physical layout of the fiber delay-line buffers is illustrated in Fig. 3. 
Furthermore we add, that since the switches are packet switches synchronous arrival of packets is needed for proper operation. Therefore, optical cell synchronizers as experimentally demonstrated in, e.g., [11] must be inserted at the input of the optical packet switches in Fig. 2.

To investigate how the buffer size and consequently the size of the space-switches in Fig. 2 is influenced by the traffic parameters, a theoretical queuing analysis is carried out.

\section{THEORY}

For the switches in Fig. 2 it is assumed that the cells arrive synchronously. This means, that in each time slot (time slot $=$ cell duration) between 0 and $n N$ cells have to be distributed to the $N$ physical outlets. Furthermore, we assume that the probability of a cell arriving at one of the $n$ wavelength channels per inlet is given by the time independent load, $\rho$. Assuming this, the probability that $k$ cells are going to a given outlet, $a_{k}$, can be found when it is assumed that a cell is equally likely to go to any outlet, i.e., the probability for the cell to go to a given outlet is $1 / N$

$$
a_{k}=\left(\begin{array}{c}
n N \\
k
\end{array}\right)\left(\frac{\rho}{N}\right)^{k}\left(1-\frac{\rho}{N}\right)^{n N-k} .
$$

The above equation comprises three factors. The first denotes the number of ways in which $k$ cells can be chosen among $n N$ cells. The second factor is the probability that $k$ cells go to the same outlet bearing in mind that a cell appears with the probability, $\rho$. Finally, the third factor is the probability that the remaining $n N-k$ cells go to other outlets.

The queues are characterized by the number of cells that can be stored $B$ and by the number of cells that can leave the queue in each time slot, $n$. Combining these characteristics with the cell arrival probabilities, $a_{k}$, enable us to write the state transition probabilities $P_{i j}$ for the number of cells in the queue

$$
P_{i j}= \begin{cases}\sum_{l=0}^{n-i} a_{l}, & 0 \leq i \leq n, j=0 \\ a_{j-i+n}, & 0 \leq i \leq n, 1 \leq j \leq B-1 \\ a_{j-i+n}, & n+1 \leq i \leq B, i-n \leq j \leq B-1 \\ \sum_{l=B-i+n}^{n N} a_{l}, & 0 \leq i \leq B, j=B \\ 0, & \text { else. }\end{cases}
$$

To clarify, $P_{i j}$, is the transition probability of having $j$ cells in the queue at the end of time slot $m$ given there were $i$ cells in the queue at the end of time slot $m-1$.

Denoting the probability for having $i$ cells $(0 \leq i \leq B)$ in the queue at the end of time slot $m$ by $q_{i}^{m}$, the following relation between the number of cells in the queue at the end of two consecutive time slots can be established

$$
\left[\begin{array}{lll}
q_{0}^{m-1} & \cdots & q_{B}^{m-1}
\end{array}\right] \underline{\underline{P}}=\left[\begin{array}{c}
q_{0}^{m} \\
\vdots \\
q_{B}^{m}
\end{array}\right]
$$

where $P_{i j}$ form the matrix, $\underline{\underline{P}}$. Eliminating the time dependency $\left(q_{i}^{m} \Rightarrow q_{i}\right)$ in (3) along with the condition: $\sum_{i=0}^{B} q_{i}=1$, form a set of $B+2$ equations with $B+1$ unknown that enables us to find $q_{i}$. This in turn allows calculation of the cell loss probability as described in the following.
For a specific outlet the average number of cells that leave the queue in a time slot is denoted, $n-\rho_{O \lambda}$, where $\rho_{O \lambda}$ is the mean number of wavelengths that are not used at the given outlet. From (1), the mean number of cells that arrive at the queue in each time slot is found to be, $n \rho$. We are now able to determine the cell success probability, CSR $=1-$ CLR (CLR is the cell loss ratio or the cell loss probability), which is the number of successfully transmitted cells, $n-\rho_{O \lambda}$, divided by the mean number of cells that enter the queue in each time slot, $n \rho$. From the distribution of cells in the queue $q_{i}$ the mean number of unused wavelengths $\rho_{O \lambda}$ can be calculated as

$$
\rho_{o \lambda}=\sum_{i=0}^{n} \sum_{j=0}^{n-i} a_{j} q_{i} \cdot(n-(i+j)) .
$$

Finally, we can write the cell loss probability (CLR)

$$
\mathrm{CLR}=1-\mathrm{CSR}=1-\frac{n-\rho_{o \lambda}}{n \rho} .
$$

When using the wavelength dimension to enhance the number of available buffer positions it should be noted, that the buffer size, $B$ does not equal the number of fiber delaylines, since each delay-line can store $n$ cells simultaneously, i.e., the number of fiber delay-lines is $B / n$.

\section{MODEL VERIFICATION AND \\ Cell Sequence Considerations}

To assess the validity of the model described above, results obtained analytically are compared to numerical simulations in Fig. 4 for a $16 \times 16$ switch with a load of 0.8 on each of the $\mathrm{n}$ wavelength channels per inlet. In the simulations the Monte Carlo method [12] is used to generate the arrival process given by (1). Evidently, the model and the simulations predict the same CLR versus the number of fiber delay-lines.

At this time a comment on the cell sequence must be made. Since the wavelength dimension is fully utilized it is possible for a cell to "catch up" and in the next network node "overtake" another cell even though the two cells entered the network a cell period or more apart. This interchange is not allowed since the cell sequence of the cells belonging to the same virtual path (VP) must be maintained. However, since cells belonging to the same VP follow the same physical path through the network they will arrive at the same inlets of the switches they pass. Therefore, it is sufficient for the switch to keep the sequence of cells arriving at the same inlets. In fact, even interchange of cells arriving at the same inlet can be allowed if the cells do not belong to the same VP. In the worst case situation where cells that address the same switch outlet and arrive the at same inlet are assumed to belong to the same VP Fig. 5 gives simulated results (filled circles) for which the cell sequence is kept. The results are compared to analytic predictions for which the order of the cells have not been kept (solid curves). The latter corresponds to the analytical values that can be found from the model given in the previous section. Fig. 5(a) is for a $4 \times 4$ switch while Fig. 5(b) is for a $16 \times 16$ switch. In both figures the load on each of the $n$ wavelength channels per inlet is 0.8. Comparison of the two sets of results shows that keeping the cell sequence even in the worst case has 


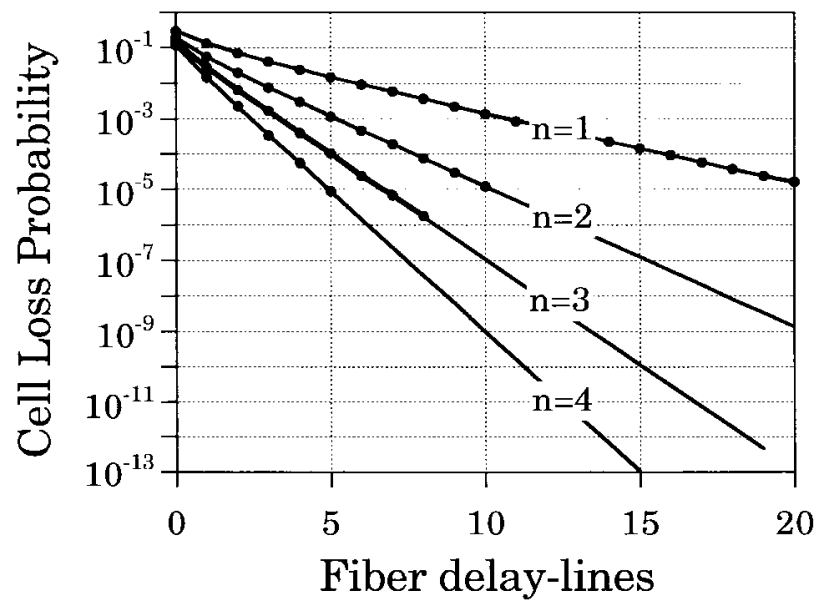

Fig. 4. Cell loss probability versus the number of fiber delay-lines, $B / n$, at a channel load of 0.8 for a $16 \times 16$ switch with one, two, three, and four wavelength channels per inlet, respectively.

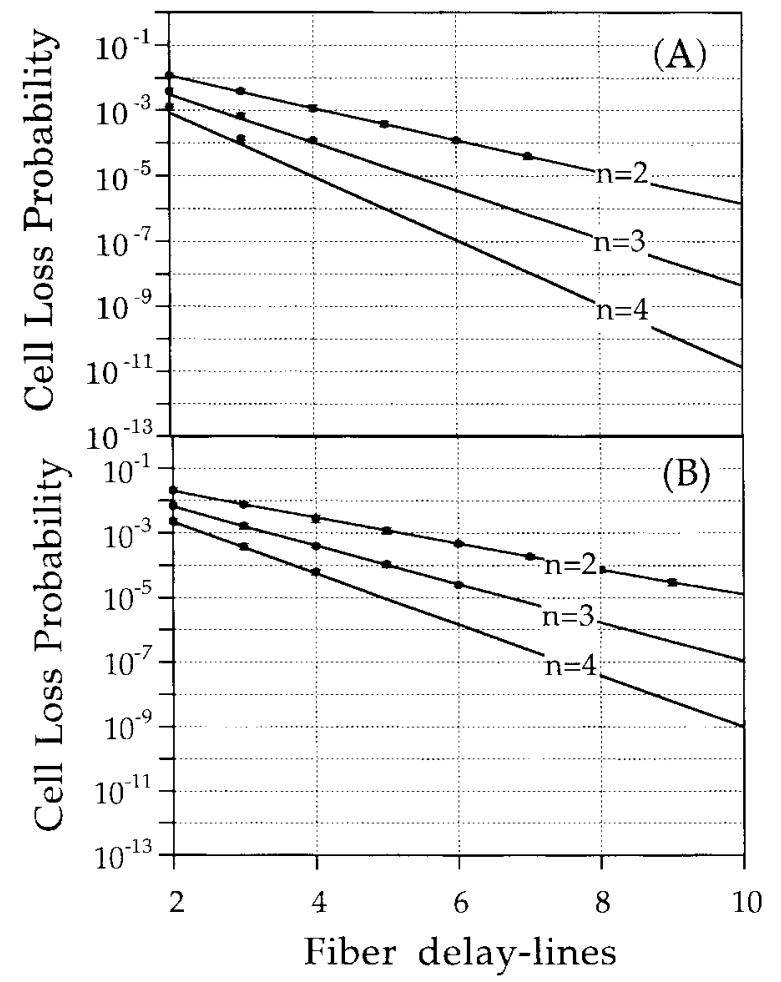

Fig. 5. Cell loss probability versus the number of fiber delay lines for a $4 \times 4$ switch (A) and a $16 \times 16$ switch (B). The number of wavelength channels per inlet, $n$, is the parameter and the load on each channel is 0.8 . The solid curves are analytical results for a buffer that does not keep the cell sequence, while dots are simulated results for a buffer that keeps the cell sequence.

little influence on the cell loss performance at least at cell loss probabilities above $10^{-5}$. Consequently the analytical model described above is used in the following analysis.

\section{INFLUENCE OF TUNEABLE WAVELENGTH CONVERTERS ON THE Number OF FIBER Delay-Lines}

As previously mentioned, tuneable wavelength converters are essential for the performance of the switch block. This is clear from Fig. 6 that illustrates the buffering process with and without tuneable wavelength converters.

Obviously, the TOWC's reduce the number of fiber delaylines. This is confirmed in Fig. 7 where the cell loss probability is shown versus the number of delay-lines, $B / n$. Results are for a $16 \times 16$ switch with a load of 0.8 for each of the $n$ wavelength channels per inlet. Without converters the performance is independent of $n$ because the queue can be viewed as consisting of $n$ separate and independent queues, each being unique for one wavelength. With converters, the switch performance in terms of the cell loss probability is improved as the number of wavelengths is increased. For comparison, 12 and six delay-lines are needed for $n$ equal to four and eight, respectively.

\section{COMPLEXITY ANALYSIS IN TERMS OF COMPONENT COUNT}

Importantly, the size of the space switch is nearly independent of the number of wavelengths. As a result, it is possible to increase the switch capacity by increasing the number of wavelength channels per in- and out-let while keeping the complexity in terms of component count almost constant. This is verified by calculating the term $N x n \cdot N \cdot \alpha$ from Fig. 2(a) for different values of $n$ (results for $n=4$ and $n=8$ can be found using Fig. 7).

In Fig. 8, the complexity is analyzed in more detail. Fig. 8(a) shows the required number of fiber delay-lines (@ CLR $=10-^{10}$ ) versus the channel load for 1,2 , and 4 wavelength channels per inlet, whereas, Fig. 8(b) shows the normalized space-switch size, $n \cdot \alpha$ for configuration $a$, (normalized with respect to the number of in- and out-lets). Although the required number of delay-lines is reduced when increasing $n$ [see Fig. 8(a)], the actual number of cells that can be stored, i.e., $n \cdot \alpha$, is nearly constant [see Fig. 8(b)]. Consequently, the size of the space switch is unaffected when the switch throughput is increased by increasing number of wavelength channels. On the other hand, Figs. 7 and 8 illustrates that the term $\alpha$ is constant and independent of the number of wavelength channels, $n$, per in- and out-let when there are no tuneable converters. This means, that if the switch size, $N$, is kept constant the space-switch size $N x n \cdot N \cdot \alpha$ grows linearly with $n$. Hence, the importance of having tuneable wavelength converters is obvious.

Another interesting aspect can be derived from Fig. 8(a). If a network consists of $16 \times 16$ switches and one wavelength channel is used per fiber $(n=1)$, Fig. 7 shows that a cell loss probability of $10^{-10}$ requires 47 delay-lines at a load of 0.8 . If four wavelength channels are used per fiber in the network this would require a channel load of 0.2 to carry the same amount of traffic. If it is possible to route data between the nodes so that the traffic is equally distributed through the network, the results in Fig. 8(a) predict that only two fiber delay-lines are needed (@CLR $=10^{-10}$ ). In fact, calculations following this principle give that for more than 11 wavelength channels no fiber delay-lines are needed. Thus, the optical buffer can be completely avoided. 


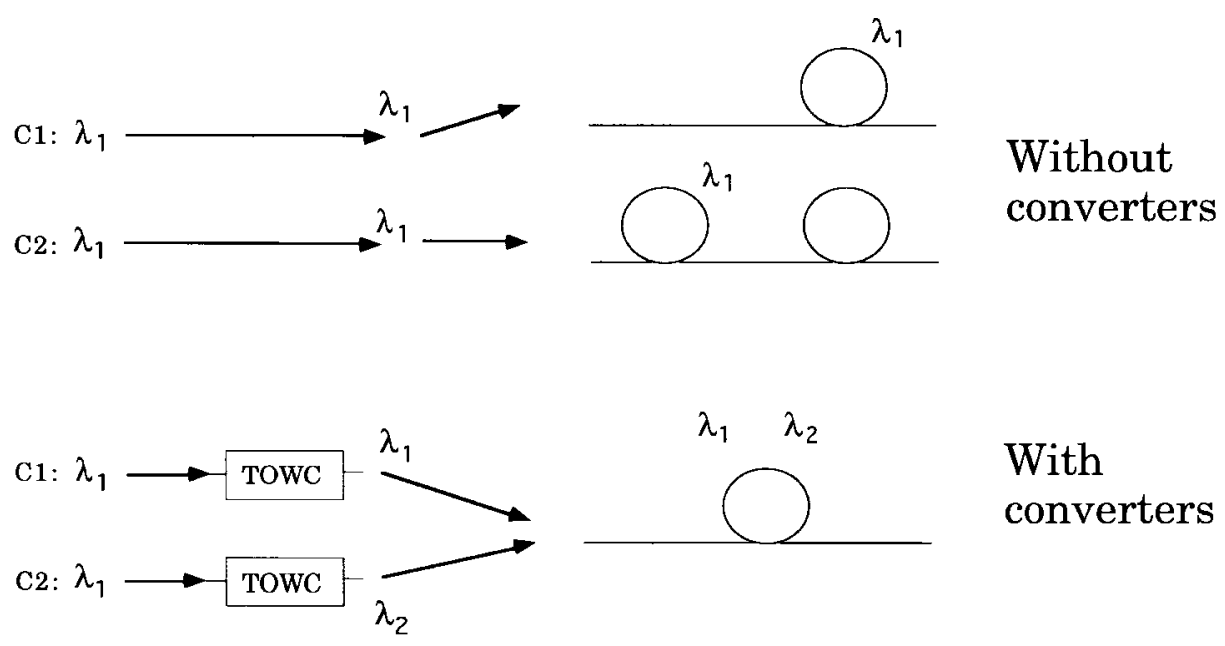

Fig. 6. Buffer filling process with and without wavelength converters. Two cells, $C 1$ and $C 2$ destined for the same outlet, arrive simultaneously at the same wavelength, $\lambda_{1}$. Without converters, two fiber delay-lines are needed to store the cells, whereas with converters, only one delay-line is needed.

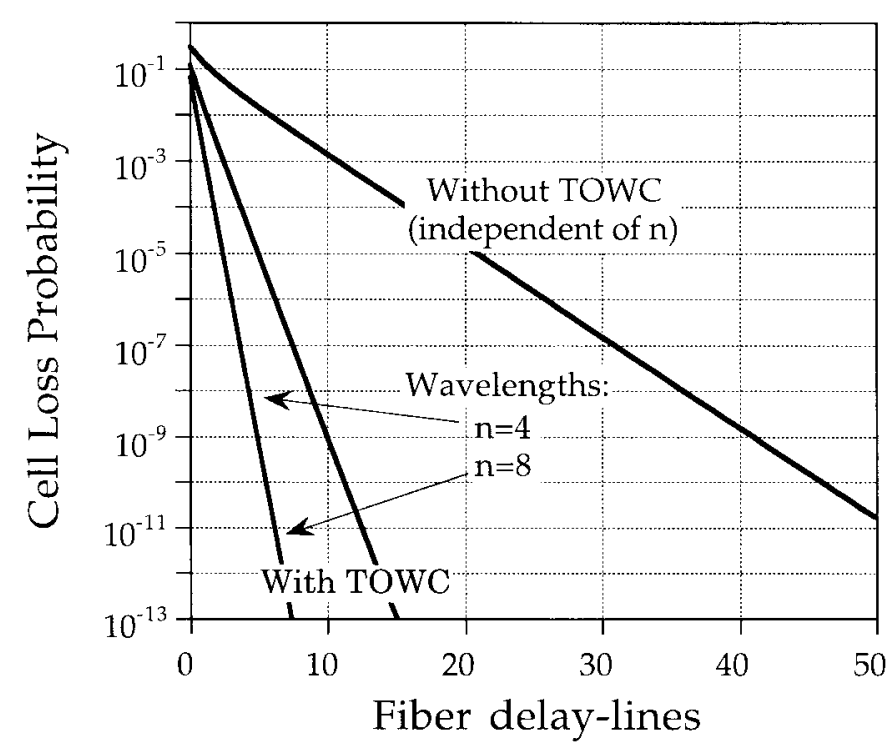

Fig. 7. Cell loss probability with and without tuneable wavelength converters versus the number of fiber delay-lines, $B / n$, at a channel load of 0.8 for a $16 \times 16$ switch with four and eight wavelengths per inlet.

The component counts for configurations $b$ and $c$, are illustrated in Fig. 9 (in the case of $n>1$ Table I gives expressions for the number of devices). The total number of cross-points or gates required to realize the space switches for $4 \times 4$ [Fig. 9(a)], $8 \times 8$ [Fig. 9(b)], and $16 \times 16$ [Fig. 9(c)] switch blocks are given versus the number of wavelength channels, $n$, per switch inlet for a channel load of 0.8. The number of cross-points are plotted for the number of fiber delay-lines that ensures a cell loss probability below $10^{-10}$. This means that the CLR is not quite the same for all points (although less than $10^{-10}$ for all) which explains the dips in the curves for some combinations of $n$ and $N$. As already concluded, the complexity of configuration $a$ is unaffected when $n$ is increased. The complexity of configuration $b$ also remains unchanged when $n$ is increased, while the complexity of configuration $c$ grows linearly with $n$. Additionally, we note that as long as the number of in- and out-lets, $N$, is smaller

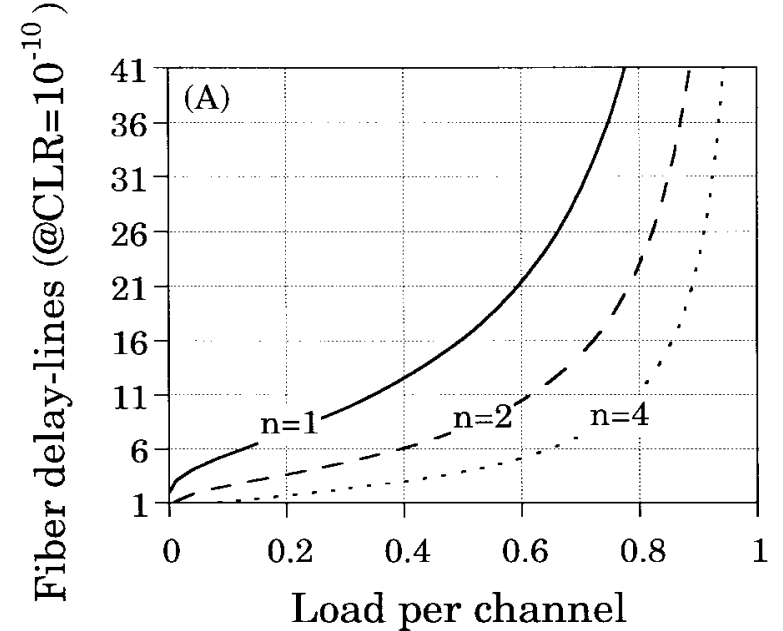

(a)

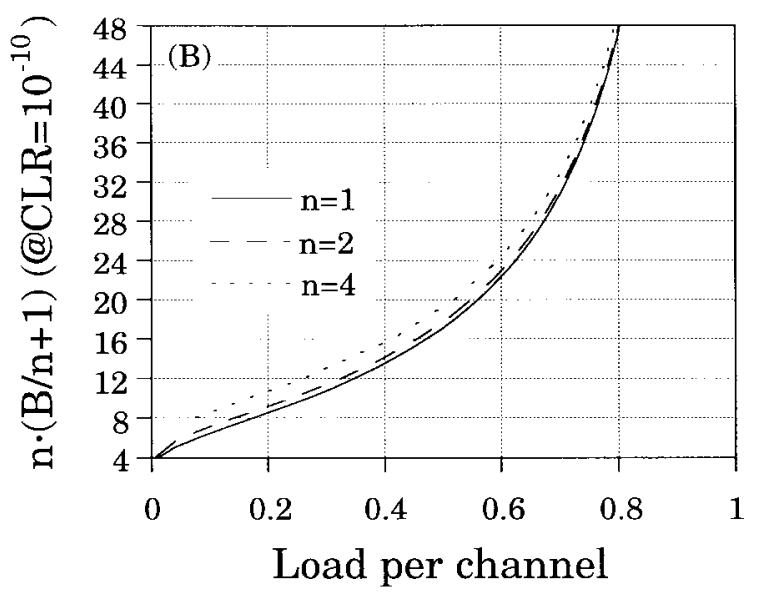

(b)

Fig. 8. (a) Required number of fiber delay lines (@CLR $=10^{-10}$ ) versus channel load for a $16 \times 16$ switch with one, two, and four wavelength channels per inlet. (b) The term $n \cdot(B / n+1)\left(@ \mathrm{CLR}=10^{-10}\right)$ versus channel load for a $16 \times 16$ switch with one, two, and four wavelength channels per inlet.

than the number of wavelengths, $n$, per in- and out-let the space-switch size required by configuration $c$ is smaller than 
for configuration $a$, while configuration $b$ is less complex in terms of the space switch size independent of $N$ and $n$.

It is important to note that the number of gates given in Fig. 9 contributes by far the most to the total component count, as can be seen from Table I. The number of gates is also the parameter which determines the differences between the three architectures when considering this type of complexity. The number of WDM multiplexers and demultiplexers is highest for configurations $b$ and $c$ while the number of tuneable wavelength converters is the same for all three configurations. Apart from the differences in the space switch sizes, configuration $b$ and $c$ require fixed wavelength converters (OWC's). The number of OWC's is on the other hand small compared to the number of gates, so the disadvantage of these two architectures is that the two wavelength conversion stages will require extra control and management.

\section{DEVICE REQUIREMENTS VERSUS SWITCH BlOCK ARCHITECTURES}

So far, we can conclude that a tradeoff exists between having as few wavelengths as possible (configuration $a$ is preferred) and as few components as possible (configuration $b$ is preferred). However, very important issues to consider for all architectures are the number of wavelengths and the wavelength sensitivity of the components involved. This will be discussed in the following.

1) Gates: Semiconductor optical amplifiers (SOA's) are attractive as gates since they are able to perform gating and amplification simultaneously. Furthermore, large $(>40 \mathrm{~dB})$ gating on/off ratios can be obtained with short switching times $(\approx 1 \mathrm{~ns})$ [13]. Moreover, they offer polarization independent on-state fiber to fiber gain of up to $29 \mathrm{~dB}$ [14]. High on/off ratios are essential for proper operation in the presence of crosstalk which is inevitable in a WDM switching environment [15]. For an interfering channel at the same wavelength as the signal an on/off ratio of approximately $25 \mathrm{~dB}$ is required to keep the power penalty (@BER $=10^{-9}$ ) below $1 \mathrm{~dB}$ [16], whereas, only about $7 \mathrm{~dB}$ is required for a crosstalk signal placed so that the beat product is well outside the receiver bandwidth [17]. The draw-back of the SOA-gate is a wavelength dependent on/off ratio [13] as well as gain saturation at high input power levels which causes the extinction ratio to collapse [18]. The wavelength dependency favors configuration $a$ featuring the lowest number of wavelengths. It should be noted that recently [19], [20], the use of gain clamped SOA's have been reported as a promising way for solving these problems, though at the expense of a lower gain. Also to be considered as gates, is the use of electro-absorption gates (EA-gates) that offer high on/off ratios $(>30 \mathrm{~dB})$ and very fast switching (up to $40 \mathrm{~Gb} / \mathrm{s}$ ) [21]). Moreover, digital switches with low loss $(\approx 4 \mathrm{~dB})$ and on/off ratios in excess of $20 \mathrm{~dB}$ [22] could be considered as gates. However, due to the loss for these devices (typically 8-10 dB for EA-gates [23]) some extra amplification is needed which in turn will add to the overall switch complexity.

2) WDM (De)multiplexers: Since crosstalk must be limited it is clear from Fig. 2 that the WDM multiplexers as well

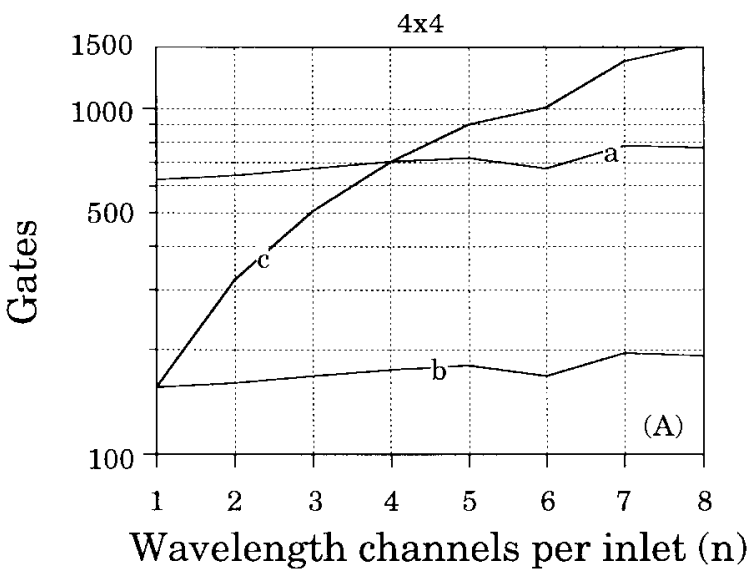

(a)

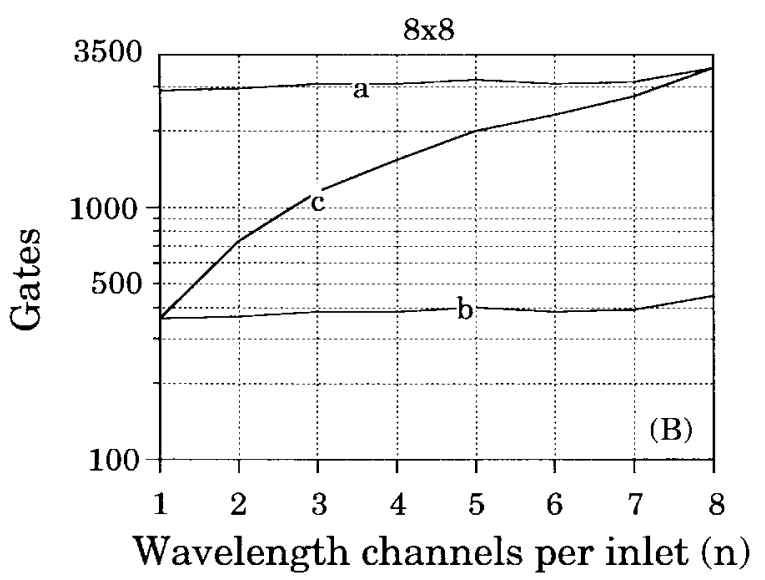

(b)

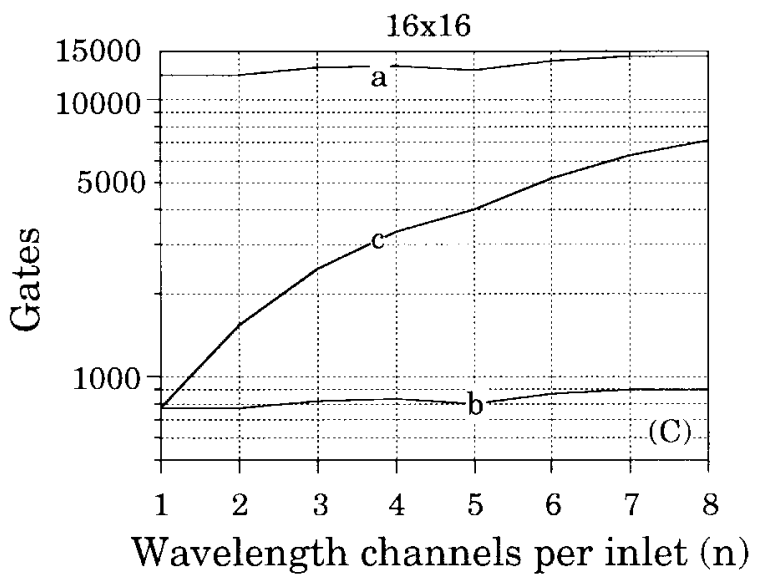

(c)

Fig. 9. Total number of gates ( $@$ CLR $=10^{-10}$ ) versus the number of wavelength channels per switch inlet, $n$. Results are for switch block configurations with four in- and out-lets (A), eight in- and out-lets (B) and 16 in- and out-lets (C). The curves are marked according to the considered configuration (see Fig. 2).

as demultiplexers must have high suppression ratios. In [24] suppression ratios larger than $20 \mathrm{~dB}$ was reported for a $8 \times 8$ demultiplexer with $0.7 \mathrm{~nm}$ spacing between the channels. Referring to the required crosstalk levels (listed above) this is sufficient to keep the crosstalk induced penalty well below $1 \mathrm{~dB}$ even if all seven channels contribute equally to the crosstalk (interferometric noise is not considered for WDM 
TABLE I

Number of DifFerent Devices for the Three ArChitectures. TOWC's ARE Tuneable Optical WaVElength CONVERTERS While OWC's ARE Optical WAVELENGTH CONVERTERS That Convert to A Fixed Output WaVelength $(n>1)$

\begin{tabular}{|l|c|c|c|}
\hline & configuration $a$ & configuration $b$ & configuration $c$ \\
\hline TOWC & $n \cdot N$ & $n \cdot N$ & $n \cdot N$ \\
\hline OWC & 0 & $n \cdot N$ & $n \cdot N$ \\
\hline WDM MUX/DMUX & $N$ & $3 \cdot N$ & $2 \cdot N+n$ \\
\hline $\begin{array}{l}\text { Space-switch } \\
\text { size (gates) }\end{array}$ & $N \times n \cdot N(B / n+1)$ & $N \times n \cdot(B / n+1)$ & $N \times n^{2} \cdot(B / n+1)$ \\
\hline
\end{tabular}

(de)multiplexers). The number of wavelengths that the passive WDM MUX/DMUX's can handle will of course determine which of the architectures is the most realistic. At the moment multiplexers and demultiplexers as large as $16 \times 16$ seem feasible [25], [26]. So the passive WDM devices will not limit the size of any of the switch blocks if the product $n \cdot N$ is kept below 16. Furthermore, typical channel spacings of 0.5 to 1 $\mathrm{nm}$ and losses of $5 \mathrm{~dB}$ for WDM MUX-DEMUX's [27] should be acceptable for most switch-block constructions. Concerning the latter, it should be noted that a 1:8 WDM demultiplexer realized by a passive splitter and filters results in a minimum loss of $10 \log (8) \approx 9 \mathrm{~dB}$ not considering filter losses.

3) Wavelength Converters: For the optical wavelength converters the most promising techniques at present is cross gain modulation (XGM) in single SOA's [28], [29] and cross phase modulation (XPM) in SOA's situated either Mach-Zehnder [30], [31] or Michelson interferometers [32].

The interferometric converters work independent of wavelength in wavelength spans covering the EDFA window. The XGM converters show some wavelength dependence especially for conversion to longer wavelengths [33] and should be used in switch configurations with a limited wavelength span. Still, this converter type as well as SOA gates were successfully used in [34] to operate a $4 \times 4$ packet switch and in [35] to demonstrate a WDM cross-connect.

The limitations for the converters could be the tuneability requirements. The larger the number of wavelengths the higher the demands on the tuneable sources delivering the signal carriers. This favors configuration $a$ since a smaller range is required. A compact method to realize the tuneability is by using one or two widely tuneable DBR- or Y-lasers to cover the EDFA window. Should the tuning speed of the lasers become a problem the straight forward method is to use a bank of lasers.

\section{CONCLUSION}

A traffic model that describes the queuing performance of an optical multiwavelength packet switch for a WDM network has been derived and verified. The influence of tuneable wavelength converters on the required number of delay-lines in the buffer has been investigated. It has been shown, that for a $16 \times 16$ switch with four and eight wavelength channels each at a load of 0.8 , the required number of delay-lines is reduced from 47 to 12 and 8, respectively, using wavelength converters. Clearly, the complexity in terms of component count of the switch architecture is greatly influenced by this reduction.
TABLE II

Comparison of Switch Architectures

\begin{tabular}{|l|c|c|c|}
\hline & Component count & Component complexity & Scalability \\
\hline Configuration $a$ & high & low & high \\
\hline Configuration $b$ & low & high & medium \\
\hline Configuration $c$ & medium & medium & low \\
\hline
\end{tabular}

Three different switch block configurations were investigated. The first switch block $(a)$ uses a physical buffer per outlet resulting in as many internal wavelengths in the switch as there are wavelengths in the network. The second switch block $(b)$ has one physical buffer completely shared among all outlets, thus requiring $n \cdot N$ wavelengths where $N$ is the number of in- and out-lets. The third configuration $(c)$ physically shares a number of buffers among all outlets and requires $N$ internal wavelengths.

Due to the difference in the number of needed wavelengths, the tuneability requirements to the wavelength converters differ as does the wavelength dependency requirements to the other components involved. However, when analysing the complexity in terms of the number of components used by each switch block, it is seen that an equal number of tuneable optical wavelength converters are needed for three switch blocks. For configurations $b$ and $c$, an equal number of fixed wavelength converters are required, whereas, the wavelength converters used for architecture $a$ are all tuneable.

The presented architectures are compared in Table II in terms of i) space-switch sizes, ii) the complexity of the involved optical devices including wavelength converters, and iii) the ability of the architectures to scale with the number of wavelength channels (more dense WDM). Regarding the component count of the space-switch, the complexity is highest for configuration $a$ with one buffer per outlet while configuration $b$ with one physically shared buffer shows the best performance. However, this is at the expense of more stringent requirements to the optical devices, since the number of internal wavelengths is higher than for configurations $a$ and $c$. As seen in the table, this results in a high complexity for configuration $b$ while requirements to the devices in configuration $a$ are the most relaxed. When increasing the number of wavelengths the switch throughput is increased. Calculations presented here, show that the total component count of the space-switches remains constant for both configuration $a$ and $b$ when tuneable converters are used to achieve efficient buffering. On the other hand, the complexity grows linearly with the number of wavelength channels for configuration $c$ thereby restricting the scalability of the switch throughput.

Without tuneable wavelength converters, the space switch grows linearly with the number of wavelengths for both configuration $a$ and $b$, while it grows with the number of wavelengths squared for configuration $c$. Therefore, tuneable wavelength converters are essential to achieve switch blocks with low complexity.

\section{REFERENCES}

[1] C. Brackett, "Dense wavelength division multiplexing networks: Principles and applications," IEEE J. Select. Areas Commun., vol. 8, pp. 948-964, Aug. 1990. 
[2] M. Sotom, A. Jourdan, P. A. Perrier, and L. Berthelon, "Photonic network and node architectures for a flexible multigigabit transport network," in Proc. ICC'93 Workshop on Optical Networks and Nodes, Geneva, Switzerland, May 1993, paper no. 2.

[3] P. Green et al., "All-optical packet-switched metropolitan-area network proposal," J. Lightwave Technol., vol. 11, pp. 754-763, May 1993.

[4] P. Dumortier et al., "Telecom networks going photonic: Reconciling transparency with scalability," in Tech. Dig. Topic. Meeting Photon. Switching'95, Salt Lake City, UT, Mar. 1995, pp. 21-23.

[5] J. M. Gabriagues and J. B. Jacob, "Exploitation of the wavelength domain for photonic switching in IBCN," in Proc. ECOC'91, Paris, France, Sept. 1991, vol. 2, pp. 59-66.

[6] M. Calzavara et al., "Optical-fiber-loop memory for multiwavelength packet buffering in ATM switching applications," in Tech. Dig. OFC'93, San Jose, CA, vol. 4, pp. 19-20, Feb. 1993.

[7] K. Wünstel et al., "Multidimensional optical switching with advanced key components," in Proc. ECOC'93, Montreux, Switzerland, Sept. 1993, vol. 2, pp. 89-92.

[8] M. G. Hluchyj et al., "Queueing performance in high-performance packet switching," IEEE J. Select. Areas Commun., vol. 6, pp. 1587-1597, Dec. 1988

[9] S. L. Danielsen et al., "A photonic WDM packet switch with reduced complexity due to wavelength converters," in Proc. Photon. Switching'95, Salt Lake City, UT, Mar. 1995, pp. 39-41.

[10] A. Y.-M. Lin and J. A. Silvester, "On the performance of an ATM switch with multichannel transmission groups," IEEE Trans. Commun. vol. 41, pp. 760-769, May 1993.

[11] M. Burzio et al., "Optical cell synchronization in an ATM optical switch," in Proc. ECOC'94, Florence, Italy, 1994, vol. 2, pp. $581-584$.

[12] White et al., Analysis of Queueing Systems. New York: Academic, 1975 , 1st ed.

[13] B. Mikkelsen et al., "Optical amplifiers as optical gates and their future applications," in Proc. EFOC/LAN'92, Invited paper, Paris, France, June 1992.

[14] P. Doussiere et al., "Polarization independent $1550 \mathrm{~nm}$ semiconductor optical amplifier packaged module with $29 \mathrm{~dB}$ fiber to fiber gain," in Tech. Dig. OAA'95, Davos, Switzerland, June 1995, pp. 119-122.

[15] J. Zhou et al., "Cross talks in multiwavelength optical cross-connect networks," in Tech. Dig. OFC'95, San Diego, CA, Feb. 1995, paper ThI8, pp. 278-280.

[16] E. L. Goldstein et al., "Performance implications of component crosstalk in transparent lightwave networks," IEEE Photon. Technol. Lett., vol. 6, pp. 657-660, May 1994.

[17] A. A. Al-Orainy and J. J. O'Reilly, "Error probability bounds and approximations for the influence of crosstalk on wavelength division multiplexed systems," Proc. Inst. Elect. Eng., vol. 137, pt. J, pp. 379-384, Dec. 1990

[18] B. Mikkelsen et al., "High performance semiconductor optical amplifiers as in-line- and pre-amplifiers," in Proc. ECOC'94, Florence, Italy, Sept. 1994, vol. 2, pp. 710-713.

[19] L. Lablonde et al., "Experimental and theoretical investigation of a gainclamped semiconductor optical amplifier," in Proc. ECOC'94, Florence, Italy, Sept. 1995, vol. 2, pp. 715-718.

[20] D. Chiaroni et al., "High performance semiconductor optical amplifier gate for fast WDM packet switching," in Proc. ECOC'95, Brussels, Sept. 1995, vol. 1, pp. 115-118.

[21] T. Ido et al., "High speed MQW electroabsorption optical modualtors integrated with low-loss waveguides," IEEE Photon. Technol. Lett., vol. 7, pp. 170-172, Feb. 1995.

[22] M. Renaud et al., "Compact digital optical switches for low insertion loss large switch arrays on InP," in Proc. ECOC'95, Brussels, Belgium, 1995, vol. 1, pp. 99-102.

[23] T. Ido et al., "An ultra-high-speed $(50 \mathrm{GHz}) \mathrm{MQW}$ electro-absorption modulator with waveguides for $40 \mathrm{Gbit} / \mathrm{s}$ modulation," in Tech. Dig. IOOC'95, Hong Kong, June 1995, vol. 5, pp. 1-2.

[24] M. Zirngibl et al., "Polarization independent $8 \times 8$ waveguide grating multiplexer on InP," Electron. Lett., vol. 29, no. 2, pp. 201-202, Jan. 1993.

[25] M. Zirngibl et al., "Demonstration of $15 \times 15$ arrayed waveguide multiplexer on InP," IEEE Photon. Technol. Lett., vol. 4, pp. 1250-1253, Nov. 1992.

[26] S. Suzuki et al., "Polarization insensitive arrayed-waveguide grating multiplexer with $\mathrm{SiO}_{2}$-on-SiO${ }_{2}$ structure," Electron. Lett., vol. 30, no. 8, pp. 642-643, Apr. 1994.

[27] B. H. Verbeek and M. K. Smit, "Phased array based WDM devices," in Proc. ECOC'95, Brussels, Belgium, Sept. 1995, vol. 1, pp. 195-202.
[28] C. Joergensen et al., " $4 \mathrm{~Gb} / \mathrm{s}$ optical wavlength conversion using semiconductor optical amplifiers," IEEE Photon. Technol. Lett., vol. 5, pp. 657-660, June 1993

[29] J. M. Wiesenfeld et al., "Bit error rate performance for wavelength conversion at $20 \mathrm{Gbit} / \mathrm{s}$," Electron. Lett., vol. 30, pp. 720-721, Apr. 1994.

[30] N. Vodjdani et al., "All optical wavelength conversion at 5 Gbit/s with monolithic integration of semiconductor optical amplifiers in a passive asymmetric Mach-Zehnder interferometer," in Proc. ECOC'94, PDP, Florence, Italy, Sept. 1994, pp. 95-98.

[31] C. Joergensen et al., "High performance wavelength conversion by next generation monolithic integrated Mach-Zehnder interferometer with SOA's," in Tech. Dig. IOOC'95, Hong Kong, June 1995, pp. 58-59.

[32] B. Mikkelsen et al., "10 Gbit/s wavelength converter realized by monolithic integration of semiconductor optical amplifiers and Michelson interferometer," in Proc. ECOC'94, Florence, Italy, Sept. 1994, vol. 4, pp. $67-70$

[33] K. E. Stubkjaer et al., "Optical wavelength converters," in Proc. ECOC'94, Florence, Italy, Sept. 1994, vol. 2, pp. 635-642.

[34] D. Chiaroni et al., "Rack-mounted $2.5 \mathrm{Gbit} / \mathrm{s}$ ATM photonic switch demonstrator," in Proc. ECOC'93, Montreux, France, Sept. 1993, Post deadline paper ThP 12.7, pp. 77-80.

[35] A. Jourdan et al., "Experimental assessment of a $4 \times 4$ four wavelength all-optical cross-connect at a $10 \mathrm{Gbit} / \mathrm{s}$ line rate," in Tech. Dig. OFC'95, San Diego, CA, Feb. 1995, paper ThI7, pp. 277-278.

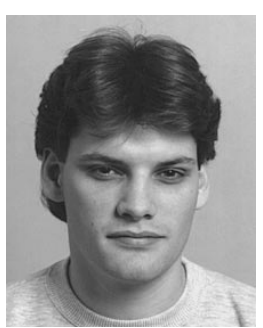

Soeren Lykke Danielsen was born in Copenhagen, Denmark, in 1970. He received the M.Sc. degree in electronic engineering from the Technical University of Denmark (TUD), Copenhagen, in 1994. He is currently working towards the Ph.D. degree at the Electromagnetics Institute.

His field of interest is high-capacity optical networks with emphasis on traffic analysis and switch blocks including optical amplifiers as functional elements.

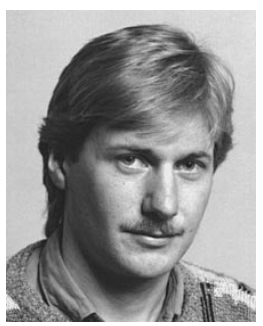

Benny Mikkelsen was born in 1960. He received the M.Sc. and Ph.D. degree from the Technical University of Denmark (TUD), Copenhagen.

$\mathrm{He}$ is currently working as a Research Associate Professor at the Electromagnetic Institute, TUD, where his fields of interest are optical amplifiers (both semiconductors and fiber type) and functional elements with emphasis on their use in systems. $\mathrm{He}$ is author and coauthor of about 100 papers and conference contributions.

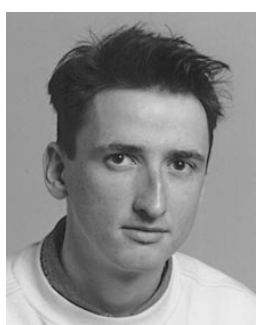

Carsten Joergensen was born in 1967. He received the M.Sc. degree in electronic engineering from the Technical University of Denmark (TUD), Copenhagen, in 1991. He is currently working towards the Ph.D. degree in optical communications at the Electromagnetics Institute, TUD.

$\mathrm{He}$ is a Research Associate at Electromagnetics Institute and his research interests include system aspects of optical amplification and processing in high-capacity optical networks. 




Terji Durhuus was born in Torshavn, Faroe Islands, in 1962, and received the M.Sc. and Ph.D. degrees from the Technical University of Denmark (TUD), Copenhagen.

Until 1995, he worked as a Research Associate at the Electromagnetics Institute, where his field of interests were optical amplifiers with emphasis on nonlinearities in semiconductor optical amplifiers.

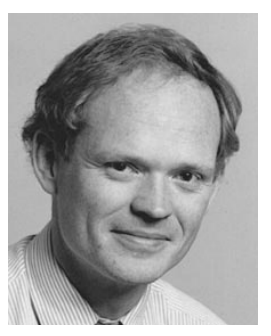

Kristian E. Stubkjaer (S'80-M'81) was born in Denmark in 1953. He received the M.Sc. and Ph.D. degrees from the Technical University of Denmark (TUD), Copenhagen, in 1977 and 1981, respectively.

From 1979 to 1981, he studied at the Tokyo Institute of Technology, Tokyo, Japan, with a scholarship from the Japanese Government. From 1981 to 1982, he was drafted for military service at the Danish Defence Research Establishment, Copenhagen. From 1982 to 1983, he was a Visiting Scientist at the IBM T. J. Watson Research Center, Yorktown Heights, NU. He is now an Associate Professor at the Electromagnetics Institute, TUD, where he is working in the field of optical communication. From 1985 to 1990, he was Director of the Electromagnetics Institute and currently he is serving as Chairman for the Electrotechnical Committee under the Technical Research Council (Danish Ministry for Education). 